\title{
GEOQUÍMICA DE RADIONUCLÍDEOS NATURAIS EM SOLOS DE ÁREAS CIRCUNVIZINHAS A UMA UNIDADE DE MINERAÇÃO E ATIVIDADE DE URÂNIO ${ }^{(1)}$
}

\author{
Gildevan Viana Cardoso(2), Nelson Moura Brasil do Amaral Sobrinho ${ }^{(3)}$, Maria \\ Angélica Vergara Wasserman ${ }^{(4)}$ \& Nelson Mazur ${ }^{(3)}$
}

\begin{abstract}
RESUMO
Os impactos ambientais advindos da exploração e do beneficiamento de U são, em grande parte, idênticos àqueles causados por atividades minero-extrativistas em geral. Este trabalho teve o objetivo de determinar a partição geoquímica dos radionuclídeos naturais ${ }^{238} \mathrm{U},{ }^{226} \mathrm{Ra}$ e ${ }^{210} \mathrm{~Pb}$ em áreas circunvizinhas à Unidade de Mineração e Atividade de Urânio (URA) das Indústrias Nucleares do Brasil S.A., localizada na Província Uranífera de Lagoa Real, no município de Caetité, na região sudoeste do Estado da Bahia. Foram coletadas amostras de solo em cinco áreas circunvizinhas à URA, representando as principais classes de solos da região, na profundidade de $0-20 \mathrm{~cm}$. Nas cinco áreas, foram determinados o teor de atividade total e o fracionamento geoquímico nas frações: F1 - levemente ácida, F2 - reduzível, F3 - oxidável, F4 - alcalina e F5 - residual. As atividades totais médias foram, em Bq kg-1 de solo, de 50 para ${ }^{238} \mathrm{U}$, 51 para ${ }^{226} \mathrm{Ra}$ e 159 para ${ }^{210} \mathrm{~Pb}$. Os extraídos na fase potencialmente biodisponível (F1) foram: $11 \%$ para ${ }^{238} \mathrm{U}, 13 \%$ para ${ }^{226} \mathrm{Ra}$ e $3 \%$ para ${ }^{210} \mathrm{~Pb} . \mathrm{O}^{238} \mathrm{U}$ apresentou maior biodisponibilidade nos solos mais ácidos e maior afinidade pelos óxidos de $\mathrm{Fe}$, o que não ocorreu para o ${ }^{226} \mathrm{Ra}$, tendo este apresentado a maior biodisponibilidade. ${ }^{210} \mathrm{~Pb}$ apresentou-se predominantemente associado a F5. As percentagens elevadas de ${ }^{238} \mathrm{U},{ }^{226} \mathrm{Ra}$ e ${ }^{210} \mathrm{~Pb}$ na fração geoquímica F5 indicam que as atividades observadas nos cinco solos estão, predominantemente, associadas ao material que deu origem a esses solos, e não a um processo de contaminação artificial em função da atividade da URA.
\end{abstract}

Termos de indexação: rádio, urânio, chumbo, partição geoquímica.

\footnotetext{
(1) Parte da Dissertação de Mestrado do primeiro autor apresentada ao Programa de Pós-Graduação em Agronomia - Ciência do Solo, Universidade Federal Rural do Rio de Janeiro - UFRRJ. Recebido para publicação em novembro de 2008 aprovado em agosto de 2009 .

(2) Engenheiro Agronomo do Instituto Nacional de Colonização e Reforma Agrária. E-mail: gildevan.cardoso@vta.incra.gov.br

(3) Professor do Instituto de Agronomia, Departamento de Solos, Universidade Federal Rural do Rio de Janeiro - UFRRJ. BR 465, km 7, CEP 23890-000 Seropédica (RJ). E-mails: nelmoura@ufrrj.br; nelmazur@ufrrj.br

(4) Professora do Instituto de Radioproteção e Dosimetria. Av. Salvador Allende s/n, Jacarepaguá, CEP 22780-160 Rio de Janeiro (RJ).E-mail: angelica@ird.gov.br
} 


\title{
SUMMARY: GEOCHEMISTRY OF NATURAL RADIONUCLIDE IN SOILS SURROUNDING A MINING AND PLANT URANIUM CONCENTRATION
}

\begin{abstract}
The environmental impacts resulting from uranium exploration and processing are to $a$ great extent identical to those caused by extractive mining activities in general. This study aimed to determine the geochemical partitioning of the natural radionuclides ${ }^{238} \mathrm{U},{ }^{226} \mathrm{Ra}$ and ${ }^{210} \mathrm{~Pb}$ in areas surrounding the Uranium Mining and Concentration Plant (URA) of the Brazilian Nuclear Industries S.A., in the uranium deposit region of Lagoa Real, in Caetité, southwestern Bahia state. Representative soil samples of the main regional soil classes were collected from the layer $0-20 \mathrm{~cm}$, in five areas around the URA. The level of total activity and geochemical fractionation (F1slightly acidic, F2 reducible, F3 oxidisable, F4 alkaline, and F5 residual) were determined for the five areas. The average total radioactivity levels were, in $B q \mathrm{~kg}^{-1}$ soil: 50 for ${ }^{238} \mathrm{U}, 51$ for ${ }^{226} \mathrm{Ra}$, and 159 for ${ }^{210} \mathrm{~Pb}$. During the potentially bioavailable phase (F1) $11 \%$ were extracted for ${ }^{238} \mathrm{U}, 13 \%$ for ${ }^{226} \mathrm{Ra}$ and $3 \%$ for ${ }^{210} \mathrm{~Pb}$. The bioavailability of ${ }^{238} \mathrm{U}$ was higher in more acidic soils and the affinity for iron oxides was greater, unlike in the case of ${ }^{226} \mathrm{Ra}$, with the greatest bioavailability. ${ }^{210} \mathrm{~Pb}$ was predominantly associated with F5. The high percentage of ${ }^{238} \mathrm{U},{ }^{226} \mathrm{Ra}$ and ${ }^{210} \mathrm{~Pb}$ in the geochemical fraction $\mathrm{F} 5$ indicates that the concentrations observed in the five soils are predominantly associated to the parent material of these soils, rather than to an artificial contamination caused by the URA activity.
\end{abstract}

Index terms: radio, uranium, lead, geochemical partitioning.

\section{INTRODUÇÃO}

Os impactos ambientais advindos da exploração e do beneficiamento de U são, em grande parte, semelhantes àqueles causados por atividades de mineração. Contudo, a presença de radioatividade no minério, rejeitos e efluentes dá origem ao impacto radiológico e, consequentemente, a aspectos adicionais relativos ao gerenciamento ambiental, como disposição e tratamento de rejeitos, controle de emissões e monitoramento ambiental (Prado, 1994). A liberação de efluentes líquidos e atmosféricos (gases e particulados) para o meio ambiente, causada pela exploração e pelo beneficiamento de U, tem o potencial de alterar a qualidade do ar, do solo e da água (Thiessen et al., 1999). Os radionuclídeos liberados na atmosfera passam por processos de mistura e dispersão e se depositam na superfície do solo e dos vegetais, entrando na cadeia alimentar (Wasserman \& Lauria, 2002). Esses processos atmosféricos são governados pelas condições de clima e topografia locais (Cardoso, 2004). A prática da irrigação também pode dar origem à contaminação dos solos e vegetais, por meio da absorção radicular e foliar de radionuclídeos, e à consequente entrada dos radionuclídeos na cadeia alimentar (IAEA, 1989).

Geralmente, os teores totais refletem as atividades desses radionuclídeos do material de origem, principalmente na fração argila (Wasserman et al., 2002b). Elementos naturalmente radioativos, como
${ }^{238} \mathrm{U},{ }^{226} \mathrm{Ra}$ e ${ }^{210} \mathrm{~Pb}$, são encontrados em minerais silicatados comuns (Eisenbud, 1987). Granitos têm usualmente várias vezes mais ${ }^{238} \mathrm{U}$ do que os basaltos. O ${ }^{226} \mathrm{Ra}$ e o ${ }^{210} \mathrm{~Pb}$ são encontrados em maiores atividades em rochas basálticas (Langmuir, 1997). Solos derivados de rochas ígneas, em geral, exibem atividades de ${ }^{238} \mathrm{U}$ típicas do material de origem, e os solos derivados de basalto, de ${ }^{226} \mathrm{Ra} \mathrm{e}^{210} \mathrm{~Pb}$ (Langmuir, 1997).

Pelo fato de esses radionuclídeos serem geralmente incorporados aos solos em uma extensão considerável, somente parte da fração incorporada é removida no curto prazo por processos geoquímicos, como migração vertical ou absorção pelas raízes das plantas. Assim, para estimar um possível risco de exposição à radiação desses radionuclídeos no longo prazo, não somente a sua atividade total no solo mas também a sua partição geoquímica, a biodisponibilidade para os processos ecológicos e os fatores que estão influenciando nessa distribuição precisam ser conhecidos (Bunzl et al., 1995; Davis et al., 1999; Butler et al., 1999).

Os oxi-hidróxidos de Fe são geralmente os mais importantes adsorventes potenciais para ${ }^{238} \mathrm{U} \mathrm{e}^{210} \mathrm{~Pb}$, com a matéria orgânica em segundo lugar (Schultz et al., 1998). O ${ }^{226} \mathrm{Ra}$ pertence ao grupo dos metais alcalinos terrosos; dessa forma, processos de adsorção e troca iônica são particularmente importantes na determinação da taxa de transporte em qualquer sistema de água subterrânea/solo. Geralmente, o ${ }^{226} \mathrm{Ra}$ 
é adsorvido por meio da formação de complexos de esfera externa, onde forças eletrostáticas são predominantes (Langmuir, 1997). Muito pouco se conhece sobre a transferência de radionuclídeos das cadeias de decaimento primordiais (radionuclídeos naturais); a maioria das investigações refere-se ao ${ }^{226} \mathrm{Ra}$, embora determinações ocasionais de ${ }^{228} \mathrm{Ra},{ }^{210} \mathrm{~Pb}$ e ${ }^{238} \mathrm{U}$ possam ocorrer (Gerzabek et al., 1998; Wasserman et al., 2000, 2002a; Wasserman \& Lauria, 2002; Ewers et al., 2003). O ${ }^{226}$ Ra é o isótopo mais importante de ocorrência na natureza, devido à sua meia-vida longa (1622 anos), associada à abundância do seu precursor $\left({ }^{238} \mathrm{U}\right)$ (Molinari \& Snodgras, 1990). Procedimentos de extração sequencial têm sido frequentemente empregados para investigar o comportamento de metais pesados e radionuclídeos artificiais no solo e sedimentos (Bourg et al., 1999; Wasserman et al., 2002a,b), porém, para o estudo do comportamento dos radionuclídeos naturais, esses procedimentos têm sido usados em poucos casos (Bunzl et al., 1995; Lima \& PennaFranca, 1998; Schultz et al., 1998).

O presente trabalho teve como objetivo avaliar as atividades de atividades totais e a distribuição geoquímica dos radionuclídeos naturais ${ }^{238} \mathrm{U},{ }^{226} \mathrm{Ra}$ e ${ }^{210} \mathrm{~Pb}$ nos solos de áreas circunvizinhas a Unidade de Mineração e Atividade de Urânio de Caetité - Ba (URA).

\section{MATERIAL E MÉTODOS}

A Unidade de Atividade de Urânio (URA) das Indústrias Nucleares do Brasil S.A. (INB) está localizada na Província Uranífera de Lagoa Real, em área pertencente ao município de Caetité, na região sudoeste do Estado da Bahia. O Complexo Mineroindustrial desenvolve atividades de pesquisa, lavra e beneficiamento de minérios nucleares, objetivando a produção de concentrado de U natural sob a forma de Diuranato de Amônio (DUA), produto usado como matéria-prima para a produção de combustível para usinas nucleares.

O clima, classificado como do tipo AW de Köppen, é tropical quente e úmido, com precipitação pluvial de aproximadamente $800 \mathrm{~mm}$ anuais e com duas estações bem definidas: seca, de maio a setembro; e úmida, de outubro a abril, quando ocorrem $80 \%$ da precipitação pluvial anual. A temperatura média é de $22^{\circ} \mathrm{C}$ e a direção predominante de ventos é a sudeste. A vegetação dominante é do tipo Caatinga, e nos pontos mais elevados ocorre Cerrado denso, de natureza semiagreste e de porte médio. As drenagens perenes são balizadas por matas-galeria, e os chapadões, cobertos pelos "campos gerais". Em grande parte da área o manto de alteração rochosa é muito espesso e fornece solos lateríticos, argilosos e arenoargilosos, grumosos, de coloração ocre. A região está relaciona- da ao conjunto de planaltos que constituem o divisor de águas da bacia hidrográfica do São Francisco, situada a uma dezena de quilômetros das jazidas, e os rios pertencem à bacia do rio de Contas, que flui para leste, em direção ao oceano Atlântico. O maciço de Caetité, onde se situam as jazidas, é limitado geomorfologicamente ao norte e ao sul, respectivamente, pelas superfícies aplainadas de Paramirim e Caculé. As altitudes variam de 750 a $1.100 \mathrm{~m}$, sendo o relevo colinoso, modelado pela ação erosiva dos rios São João e Paramirim.

A área circunvizinha da URA considerada neste estudo abrange um raio de $2 \mathrm{~km}$ a partir do centro do empreendimento. A determinação desse espaço foi necessária para definir uma área de monitoramento por parte do empreendedor, a fim de se obter um "background" radiológico que permitisse um controle sistemático do ambiente quanto ao aumento da liberação de radionuclídeos em decorrência da extração e processamento do minério e do beneficiamento do urânio pela usina.

As amostras de solo foram coletadas em cinco subáreas circunvizinhas à URA, de forma a caracterizar as classes de solos mais representativas da área sob influência da usina e que também fazem parte do Programa de Monitoração Ambiental desenvolvido pelo operador. As amostras foram coletadas na profundidade de $0-20 \mathrm{~cm}$, de maior influência da atividade agrícola, com um trado-sonda de $45 \mathrm{~mm}$ de diâmetro, retirando-se 50 amostras simples por ha para obtenção da amostra composta. Em cada área, foram obtidas três amostras compostas (Quadro 1 e Figura 1).

As amostras de terra fina seca ao ar (TFSA) foram encaminhadas aos laboratórios de Física do Solo e de Fertilidade do Solo da UFRRJ, para análise granulométrica e química, de acordo com o método da Embrapa (1997) (Quadro 2). O fracionamento geoquímico dos radionuclídeos foi realizado por meio de um procedimento de extração sequencial (Quadro 3), mediante uma adaptação do método descrito por Wasserman et al. (2001a).

Quadro 1. Localização, classes de solo, distância dos locais de coleta de amostra em relação à cava da mina e à unidade de processamento do urânio das cinco áreas de coleta de amostras de solo

\begin{tabular}{lrl}
\hline Subáreas & Distância & \multicolumn{1}{c}{ Classe de solo } \\
\hline & \multicolumn{1}{c}{ m } & \\
Ponto 8 & 941 & Latossolo Amarelo distrófico (LA) \\
Ponto 10 & 592 & Cambissolo Háplico tb CH) \\
Ponto 13 & 268 & Argissolo Vermelho-Amarelo tb (AV) \\
Ponto 42 & 2.113 & Neossolo Regolítico (NR) \\
Ponto 46 & 1.401 & Neossolo Quartzarênico A fraco (NQ) \\
\hline
\end{tabular}




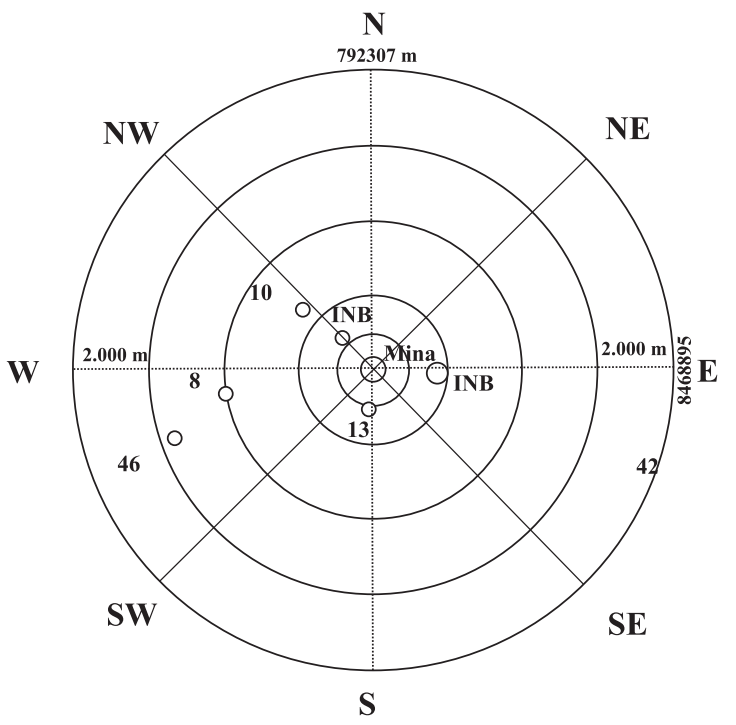

Figura 1. Esquema georreferenciado, indicando a distribuição e localização dos pontos de coleta de amostras de solo e vegetais, em relação à cava da mina, à unidade de britagem e processamento do minério (INB) e à direção predominante dos ventos (de SE para NW).
As análises para determinação das atividades totais de ${ }^{238} \mathrm{U},{ }^{226} \mathrm{Ra}$ e ${ }^{210} \mathrm{~Pb}$ e para o fracionamento geoquímico foram realizadas em triplicata. Os extratos obtidos pela extração sequencial e pela abertura total das amostras de solo foram analisados por espectrofotometria gama, com o uso de detector semicondutor de Germânio (GL2020R) de alta pureza, segundo os procedimentos radioquímicos descritos no Manual de Métodos Analíticos do IRD/DEPRA (1994). Para validação do método analítico utilizou-se um material certificado (sedimento de referência IAEA300) e foram observados valores muito próximos aos dos certificados com baixo desvio-padrão.

\section{RESULTADOS E DISCUSSÃO}

A atividade média total de ${ }^{238} \mathrm{U}$ nas cinco subáreas selecionadas foi de $50 \mathrm{~Bq} \mathrm{~kg}^{-1}$, variando de 40 a $72 \mathrm{~Bq} \mathrm{~kg}^{-1}$ (Quadro 4). O valor mais elevado de $72 \mathrm{~Bq} \mathrm{~kg}^{-1}$ foi observado no ponto 13 . Para o ${ }^{226} \mathrm{Ra}$ e o ${ }^{210} \mathrm{~Pb}$, os maiores valores foram de 74 e $196 \mathrm{~Bq} \mathrm{~kg}^{-1}$, respectivamente, e também foram verificados no ponto 13 (Quadros 5 e 6). Entretanto, os menores

Quadro 2. Características químicas e físicas ${ }^{(1)}$ dos cinco solos selecionados de áreas circunvizinhas à URA

\begin{tabular}{|c|c|c|c|c|c|c|c|c|}
\hline Local & Classe de solo & $\mathbf{p H}$ & $\mathbf{C}$ & Areia & Silte & Argila & Fe total & CTC \\
\hline & & & & & - $\mathrm{g} \mathrm{kg}$. & & & $\mathrm{cmol}_{\mathrm{c}} \mathrm{dm}^{-3}$ \\
\hline 8 & Latossolo Amarelo distrófico & 4,9 & 8 & 580 & 60 & 360 & 30,2 & 7,3 \\
\hline 10 & Cambissolo Háplico Tb & 5,8 & 8 & 790 & 90 & 120 & 10,3 & 4,0 \\
\hline 13 & Argissolo Vermelho-Amarelo & 5,1 & 11 & 600 & 80 & 320 & 45,2 & 6,6 \\
\hline 42 & Neossolo Regolítico & 6,2 & 6 & 690 & 100 & 210 & 9,8 & 5,6 \\
\hline 46 & Quartzarênico A fraco & 6,0 & 6 & 860 & 50 & 90 & 3,8 & 3,6 \\
\hline
\end{tabular}

(1) Análises granulométricas e análises químicas, de acordo com método da Embrapa (1997). pH: $\mathrm{pH}$ em água; C: carbono orgânico; Fe total: ferro total; CTC: capacidade de troca catiônica

Quadro 3. Descrição do procedimento de extração sequencial dos radionuclídeos ${ }^{(1)}$

Amostra de 50 g solo ${ }^{(1)}$

F1: Fase levemente ácida: elementos potencialmente biodisponíveis.

Adição de $500 \mathrm{~mL}$ de $\left[\left(\mathrm{CH}_{3} \mathrm{COOH}\left(2 \mathrm{~mol} \mathrm{~L}^{-1}\right)+\mathrm{CH}_{3} \mathrm{COONa}\left(2 \mathrm{~mol} \mathrm{~L}^{-1}\right)\right.\right.$ 1:1) pH 4,7]/ Agitação por $16 \mathrm{~h} / \mathrm{Filtragem} \mathrm{a}$

vácuo com papel-filtro (5B 100/125 mm) e utilização do resíduo sólido na próxima extração.

F2: Fase redutível: elementos associados a óxidos de $\mathrm{Mn}$.

No resíduo sólido, adição de $500 \mathrm{~mL}$ de $\left[\left(\mathrm{NH}_{2} \mathrm{OH} \cdot \mathrm{HCl}-0,1 \mathrm{~mol} \mathrm{~L}{ }^{-1}\right)\right.$ acidificado com $\mathrm{HNO}_{3}$ para pH 2] / Agitação por $16 \mathrm{~h}$

Filtragem a vácuo com papel-filtro (5B 100/125 mm).

F3: Fase oxidável:elementos associados à matéria orgânica.

No resíduo sólido, adição de $300 \mathrm{~mL}$ de $\left[\mathrm{H}_{2} \mathrm{O}_{2}(30 \%)\right.$ acidificado com $\mathrm{HNO}_{3}$ para pH 2] / Agitação por 16 h / Filtragem a vácuo com papel-filtro (5B 100/125 mm).

F4: Fase alcalina: elementos associados a óxidos de Fe.

No resíduo sólido, adição de $500 \mathrm{~mL}$ de $\left[\left(\mathrm{NaOH} 0,1 \mathrm{~mol} \mathrm{~L}^{-1}\right) \mathrm{pH} 12\right]$ / Agitação por 16 h / Filtragem a vácuo com papel-filtro (5B 100/125 mm).

F5: Fase residual: elementos associados a compostos resistentes do solo.

$=[$ Total $-(\mathrm{F} 1+\mathrm{F} 2+\mathrm{F} 3+\mathrm{F} 4)]$.

Total: Abertura total de $1 \mathrm{~g}$ de solo com água régia $+\mathrm{HF}$ em chapa aquecedora (a aproximadamente $80{ }^{\circ} \mathrm{C}$ ).

(1) Adaptado de Wasserman et al. (2001a). 
valores de ${ }^{238} \mathrm{U},{ }^{226} \mathrm{Ra}$ e ${ }^{210} \mathrm{~Pb}$ foram observados nos pontos 10 e 46 .

Conforme verificado (Quadro 1), o ponto 13 é o mais próximo da cava da mina e da unidade de processamento do urânio, e o 46, o mais distante. Pelo fato de a deposição atmosférica ser uma importante via de impacto radiológico para essa região semiárida (Cardoso, 2004), possivelmente, a proximidade da cava de mineração influenciou na atividade desses radionuclídeos naturais na camada superficial do solo.

Não se pode também descartar a possibilidade de os solos Cambissolo Háplico tb, do ponto 10, e Neossolo Quartzarênico, do ponto 46, por apresentarem textura mais arenosa (Quadro 2) na camada superficial, terem atividades totais menores. Geralmente, as atividades totais refletem as atividades desses radionuclídeos do material de origem, principalmente na fração argila (Wasserman, 2002b). Elementos naturalmente radioativos são, geralmente, encontrados em minerais silicatados comuns.
A atividade média de ${ }^{238} \mathrm{U}$, nas amostras dos cinco solos analisados, na fase F1 (fração biodisponível) foi de $6 \mathrm{~Bq} \mathrm{~kg}^{-1}$ equivalendo a $11 \%$ da atividade total, variando de 7 a $20 \%$ da atividade total (Quadro 4). A maior atividade observada de ${ }^{238} \mathrm{U}$, na fração $\mathrm{F} 1$ (fração biodisponível), foi de $9 \mathrm{~Bq} \mathrm{~kg}^{-1}$ no ponto 8, no Latossolo Amarelo distrófico, equivalendo a $20 \%$ da atividade total, e no ponto 13, no Argissolo Vermelho-Amarelo. Esses dois solos são os que apresentam os maiores teores de argila e os menores valores de pH (Quadro 2). Não se pode desconsiderar uma possível interferência do pH na solubilização de particulados, possivelmente, depositados na superfície dos solos nos pontos 8 (intermediário) e 13 (o mais próximo da usina). O contato desses particulados com a maior acidez nesses solos poderia solubilizar parte desse material, que ficaria em equilíbrio com a fração trocável e, dessa forma, explicaria os percentuais mais elevados na F1.

O U ocorre nos estados de oxidação U(IV), U(V) e U(VI) e, sob condições de oxidação, encontra-se,

Quadro 4. Atividade total e obtida nas frações geoquímicas F1, F2, F3, F4 e F5 de ${ }^{238} \mathrm{U}$, na profundidade de 0 $20 \mathrm{~cm}$, nos solos das cinco áreas selecionadas

\begin{tabular}{|c|c|c|c|c|c|c|c|c|c|c|c|}
\hline \multirow{2}{*}{$\begin{array}{r}\text { Local } \\
\\
8\end{array}$} & \multirow{2}{*}{$\begin{array}{l}\text { Total } \\
44 \pm 5^{(1)}\end{array}$} & \multicolumn{2}{|c|}{ F1 } & \multicolumn{2}{|l|}{ F2 } & \multicolumn{2}{|l|}{ F3 } & \multicolumn{2}{|c|}{ F4 } & \multicolumn{2}{|c|}{ F5 } \\
\hline & & $9 \pm 2$ & $(20)^{(2)}$ & $1 \pm 0$ & (1) & $1 \pm 0$ & (2) & $2 \pm 0$ & (5) & $31 \pm 5$ & (72) \\
\hline 10 & $40 \pm 6$ & $4 \pm 1$ & (9) & $1 \pm 0$ & (2) & $1 \pm 0$ & (2) & $2 \pm 0$ & (4) & $32 \pm 4$ & (83) \\
\hline 13 & $72 \pm 8$ & $9 \pm 2$ & (12) & $1 \pm 0$ & (2) & $1 \pm 0$ & (4) & $15 \pm 1$ & (18) & $46 \pm 3$ & (63) \\
\hline 42 & $51 \pm 5$ & $4 \pm 1$ & (8) & $1 \pm 0$ & (1) & $1 \pm 0$ & (1) & nd & (1) & $45 \pm 2$ & (89) \\
\hline 46 & $43 \pm 4$ & $3 \pm 1$ & (7) & $1 \pm 0$ & (2) & $1 \pm 0$ & (2) & $2 \pm 0$ & (4) & $36 \pm 4$ & (85) \\
\hline
\end{tabular}

${ }^{(1)}$ Desvio-padrão. ${ }^{(2)}$ Os números entre parênteses representam a percentagem do total. F1: Fase levemente ácida: elementos potencialmente biodisponíveis; F2: Fase redutível: elementos associados a óxidos de Mn; F3: Fase oxidável: elementos associados à matéria orgânica; F4: Fase alcalina: elementos associados a óxidos de Fe; F5: Fase residual: elementos associados a compostos resistentes do solo.

Quadro 5. Atividade total e obtida nas frações geoquímicas F1, F2, F3, F4 e F5 de ${ }^{226}$ Ra, na profundidade de 0-20 cm, nos solos das cinco áreas selecionadas

\begin{tabular}{|c|c|c|c|c|c|c|c|c|c|c|c|}
\hline \multirow{2}{*}{$\begin{array}{c}\text { Local } \\
\\
8\end{array}$} & \multirow{2}{*}{$\begin{array}{l}\text { Total } \\
58 \pm 6^{(1)}\end{array}$} & \multicolumn{2}{|c|}{ F1 } & \multicolumn{2}{|l|}{ F2 } & \multicolumn{2}{|l|}{ F3 } & \multicolumn{2}{|c|}{ F4 } & \multicolumn{2}{|l|}{ F5 } \\
\hline & & $10 \pm 2$ & $(17)^{(2)}$ & $4 \pm 1$ & (7) & $4 \pm 1$ & (7) & nd & (0) & $40 \pm 4$ & (69) \\
\hline 10 & $34 \pm 4$ & $5 \pm 1$ & (15) & $5 \pm 1$ & (14) & $2 \pm 0$ & (8) & nd & (0) & $21 \pm 3$ & (63) \\
\hline 13 & $74 \pm 6$ & $9 \pm 1$ & (12) & $5 \pm 0$ & (7) & $11 \pm 2$ & (15) & nd & (0) & $49 \pm 5$ & (66) \\
\hline 42 & $60 \pm 4$ & $6 \pm 1$ & (10) & $6 \pm 1$ & (10) & $2 \pm 0$ & (5) & nd & (0) & $45 \pm 3$ & (75) \\
\hline 46 & $29 \pm 3$ & $5 \pm 0$ & (18) & $5 \pm 2$ & (17) & $3 \pm 0$ & (9) & nd & $(0)$ & $16 \pm 2$ & (56) \\
\hline
\end{tabular}

(1) Desvio-padrão. ${ }^{(2)}$ Os números entre parênteses representam a percentagem do total. F1: Fase levemente ácida: elementos potencialmente biodisponíveis; F2: Fase redutível: elementos associados a óxidos de Mn; F3: Fase oxidável: elementos associados à matéria orgânica; F4: Fase alcalina: elementos associados a óxidos de Fe; F5: Fase residual: elementos associados a compostos resistentes do solo. 
Quadro 6. Atividade de ${ }^{210} \mathrm{~Pb}$ total e obtida nas frações geoquímicas $\mathrm{F} 1, \mathrm{~F} 2, \mathrm{~F} 3, \mathrm{~F} 4 \mathrm{e}$ F5 de ${ }^{210} \mathrm{~Pb}$, na profundidade de 0-20 cm, nos solos das cinco áreas selecionadas

\begin{tabular}{|c|c|c|c|c|c|c|c|c|c|c|c|}
\hline Local & Total & \multicolumn{2}{|l|}{ F1 } & F2 & & \multicolumn{2}{|l|}{ F3 } & \multicolumn{2}{|l|}{ F4 } & \multicolumn{2}{|l|}{ F5 } \\
\hline 8 & $150 \pm 5$ & $7 \pm 2^{(1)}$ & $(5)^{(2)}$ & $3 \pm 1$ & (2) & $1 \pm 0$ & (1) & $10 \pm 2$ & (7) & $129 \pm 5$ & (86) \\
\hline 10 & $142 \pm 4$ & $6 \pm 1$ & (4) & $3 \pm 1$ & (2) & $3 \pm 1$ & $(2)$ & $1 \pm 0$ & (1) & $128 \pm 4$ & (91) \\
\hline 13 & $195 \pm 5$ & $6 \pm 1$ & (3) & $4 \pm 1$ & $(2)$ & $2 \pm 0$ & (1) & $15 \pm 2$ & (8) & $168 \pm 4$ & (86) \\
\hline 42 & $157 \pm 6$ & $2 \pm 0$ & (1) & $3 \pm 0$ & $(2)$ & $2 \pm 0$ & (1) & $2 \pm 1$ & (1) & $149 \pm 5$ & (95) \\
\hline 46 & $148 \pm 4$ & $4 \pm 1$ & (1) & $4 \pm 1$ & $(2)$ & $2 \pm 0$ & (1) & $2 \pm 0$ & (1) & $136 \pm 5$ & (95) \\
\hline
\end{tabular}

${ }^{(1)}$ Desvio-padrão. ${ }^{(2)}$ Os números entre parênteses representam a percentagem do total. F1: Fase levemente ácida: elementos potencialmente biodisponíveis; F2: Fase redutível: elementos associados a óxidos de Mn; F3: Fase oxidável: elementos associados à matéria orgânica; F4: Fase alcalina: elementos associados a óxidos de Fe; F5: Fase residual: elementos associados a compostos resistentes do solo.

predominantemente, na forma de íon uranila $\left(\mathrm{UO}_{2}{ }^{+2}\right)$, solúvel, formando complexos de esfera externa na superfície das argilas silicatadas e óxidos de $\mathrm{Fe}$ e $\mathrm{Al}$ (Langmuir, 1997). A menor atividade verificada no ponto 46 de $3 \mathrm{~Bq} \mathrm{~kg}^{-1}$, no Neossolo Quartzarênico A fraco, pode ter sido influenciada pela textura mais arenosa observada na camada superficial desse solo (Quadro 2).

A atividade de ${ }^{238} \mathrm{U}$ extraído na fase $\mathrm{F} 1$ (fração biodisponível), nessas cinco áreas, apresenta-se correlacionado negativamente com $\mathrm{pH}$ do solo $(\mathrm{r}=-0,91$; $p=0,029)$, o que pode ser explicado pelo aumento da solubilidade dos minerais de U, com a diminuição do pH do solo (Langmuir, 1997), como também da solubilização de particulados provenientes da usina, como discutido anteriormente. Para esse autor, nos solos de ambientes oxidados com baixos valores de $\mathrm{pH}$ (abaixo de pH 5), haverá maior solubilização dos minerais de urânio, particularmente carnotita $\left[\mathrm{K}_{2}\left(\mathrm{UO}_{2}\right)_{2}\left(\mathrm{VO}_{4}\right)_{2}\right]$ e tyuyamunita $\left[\mathrm{Ca}\left(\mathrm{UO}_{2}\right)_{2}\left(\mathrm{VO}_{4}\right)_{2}\right]$, e aumento da atividade de $\mathrm{U}$ nas frações geoquímicas mais lábeis - fato esse que pode explicar os maiores percentuais de ${ }^{238} \mathrm{U}$ obtidos para a fase $\mathrm{F} 1$ (fração biodisponível). O Argissolo Vermelho-Amarelo, no ponto 13, e o Latossolo Amarelo distrófico, no ponto 8, são os solos que apresentam os menores valores de pH (Quadro 2).

As frações geoquímicas F2 (redutível) e F3 (oxidável) foram as que mostraram as menores atividades de ${ }^{238} \mathrm{U}$ extraídas, sendo de $1 \mathrm{~Bq} \mathrm{~kg}^{-1}$ para os cinco solos nas duas frações (Quadro 2). Embora o solo Argissolo Vermelho-Amarelo seja o de maior teor de C (Quadro 1), a atividade de ${ }^{238} \mathrm{U}$ na fração associada à matéria orgânica (F3) também foi muito baixa. Esse resultado demonstra que esse radionuclídeo apresenta maior associação com a fração mineral do solo (Schultz et al., 1998).
As atividades verificadas na fração associada aos óxidos de $\mathrm{Fe}$ (F4), de maneira geral, também foram baixas, exceto no ponto 13, no Argissolo VermelhoAmarelo, onde foi observada atividade de $15 \mathrm{~Bq} \mathrm{~kg}^{-1}$. A percentagem mais elevada na F4 (18 \%), no ponto 13 , pode ser explicada pela maior ocorrência dos óxidos, hidróxidos e oxi-hidróxidos de Fe (Quadro 2). O forte comportamento adsorvente para íons uranila [U(VI)] na superfície desses minerais secundários tem sido observado (Langmuir, 1997; Ewers et al., 2003).

A fração residual (F5) foi predominante em todas as áreas, com um percentual médio de $78 \%$, variando entre 63 e $89 \%$. O fato de esses elementos estarem associados à fração mais recalcitrante do solo (F5), ou seja, à estrutura cristalina dos minerais primários e secundários e oclusa nos óxidos de Fe, demonstra que, possivelmente, os radionuclídeos analisados sejam advindos do material que deu origem a esses solos, e não de um processo de contaminação artificial decorrente da atividade da URA.

A atividade total e o fracionamento geoquímico do ${ }^{226} \mathrm{Ra}$ nos solos das cinco áreas selecionadas, na profundidade de $0-20 \mathrm{~cm}$, são apresentados no quadro 5. A atividade total média de ${ }^{226} \mathrm{Ra}$ foi de $51 \mathrm{~Bq} \mathrm{~kg}^{-1}$, variando de 29 a $74 \mathrm{~Bq} \mathrm{~kg}^{-1}$. O menor valor foi observado no ponto 46, e o maior, no 13.

A fração F1 (fração biodisponível) variou de 5 a $10 \mathrm{~Bq} \mathrm{~kg}^{-1}$, e as maiores atividades foram verificadas nos pontos 8 e 13: 10 e $9 \mathrm{~Bq} \mathrm{~kg}^{-1}$, respectivamente. O Latossolo Amarelo distrófico e o Argissolo VermelhoAmarelo têm a camada superficial com textura mais argilosa e maior CTC e os menores valores de $\mathrm{pH}$ (Quadro 2). Da mesma forma que observado para o ${ }^{238} \mathrm{U}$, os maiores percentuais na fração $\mathrm{F} 1$ nos pontos 8 e 13 podem ter sido causados pela solubilização dos particulados depositados na superfície desses dois solos mais ácidos. O ${ }^{226} \mathrm{Ra}$ é um metal alcalino terroso 
adsorvido, predominantemente, por interações eletrostáticas, na forma não específica, por meio da formação de complexos de esfera externa (Schultz et al., 1998). Dessa forma, uma vez fora da estrutura cristalina dos minerais do solo, ele apresentará forte tendência para adsorção e troca iônica, estando em equilíbrio com a solução do solo.

A fração F2 (redutível) apresentou atividades de ${ }^{226} \mathrm{Ra}$ muito próximas às da fase $\mathrm{F} 1$ (fração biodisponível), com uma média de $5 \mathrm{~Bq} \mathrm{~kg}{ }^{-1}$, variando entre 7 e $17 \%$ do total (Quadro 5).

Na fase F3 (levemente oxidável), as atividades foram menores, com uma média de $4 \mathrm{~Bq} \mathrm{~kg}^{-1}$, variando entre 5 e $15 \%$ do teor total de ${ }^{226} \mathrm{Ra}$, destacando-se apenas o ponto 13 , com a atividade de $11 \mathrm{~Bq} \mathrm{~kg}^{-1}$. A maior atividade na F3 deve estar associada ao maior teor de C do Argissolo Vermelho-Amarelo do ponto 13 (Quadro 2). Lima \& Penna Franca (1998) verificaram percentuais de ${ }^{226}$ Ra de $12 \%$ na fração oxidável (ligado à matéria orgânica) - valor próximo ao observado no ponto 13.

Na fase F4 (alcalina) o ${ }^{226} \mathrm{Ra}$ não foi detectado. Com base nos resultados obtidos nessa fase, verificase que o ${ }^{226} \mathrm{Ra}$ apresenta baixa afinidade pelos óxidos de Fe, o que, possivelmente, deve estar associado à baixa tendência dos metais alcalinos terrosos em formarem complexos de esfera interna com as hidroxilas funcionais na superfície dos óxidos de Fe, sendo o rádio o que apresenta a menor tendência entre os metais alcalinos terrosos (Molinari \& Snodgras, 1990).

A fração F5 foi predominante, variando entre $16 \mathrm{e}$ $49 \mathrm{~Bq} \mathrm{~kg}^{-1}$, com percentuais entre 56 e $76 \%$ do total. De forma análoga ao verificado para o $\mathrm{U}^{238}$, as maiores atividades também foram encontradas no ponto 13. Resultados semelhantes foram encontrados por Lima \& Penna Franca (1998), que estudaram a partição geoquímica de ${ }^{226} \mathrm{Ra}$ em solos de quatro fazendas do planalto de Poços de Caldas-MG, utilizando uma adaptação do método de extração sequencial de Tessier et al. (1979), e verificaram que os percentuais médios para o ${ }^{226} \mathrm{Ra}$, que ocorria naturalmente nos solos, foram de $59 \%$ para a fração residual.

Baeza et al. (1996), estudando a biodisponibilidade e a transferência solo-planta de radionuclídeos naturais num ecossistema do Mediterrâneo (caracterizado pela alternância das estações seca e úmida) próximo a uma usina nuclear na província de Cárceres, na Espanha, relataram que a atividade de ${ }^{226} \mathrm{Ra}$ na fração residual (F5) é a predominante, observando percentuais superiores a $60 \%$, praticamente constante dentro dos primeiros $20 \mathrm{~cm}$ dos solos estudados, o que também foi observado nos cinco solos de Caetité.

A relação ${ }^{238} \mathrm{U} / 226 \mathrm{Ra}$, com base no teor total médio desses elementos, foi de aproximadamente 1 , variando de 0,75 no ponto 8 (Latossolo Amarelo) a 1,48 no ponto 46 (Neossolo Quartzarênico A fraco), demonstrando que esses dois radionuclídeos encontram-se em equilíbrio secular, o que se justifica por se tratar de amostras ambientais, cujos radionuclídeos analisados são advindos, provavelmente, do material que deu origem a esses solos, e não de um processo de contaminação artificial em função da atividade da URA.

Nos solos das cinco áreas selecionadas para a extração das cinco frações geoquímicas, na profundidade de $0-20 \mathrm{~cm}$, a atividade média de ${ }^{210} \mathrm{~Pb}$ extraído na fase F1(fração biodisponível) foi de $5 \mathrm{~Bq} \mathrm{~kg}^{-1}$, variando de 2 a $7 \mathrm{~Bq} \mathrm{~kg}^{-1}$, equivalendo de 1 a $5 \%$ do total (Quadro 6). Os pequenos percentuais de ${ }^{210} \mathrm{~Pb}$ na $\mathrm{F} 1$ (fração biodisponível) têm sido observados em alguns trabalhos e estão relacionados com a baixa mobilidade e biodisponibiliddae desse radionuclídeo (Köhler et al., 2000; Ewers et al., 2003). Os resultados de baixa biodisponibilidade obtidos para ${ }^{210} \mathrm{~Pb}$ em Caetité estão de acordo com os obtidos por Vasconcellos et al. (1987) em solos de 14 fazendas circunvizinhas à mina de urânio de Poços de Caldas-MG. Eles relataram que a atividade total média de ${ }^{210} \mathrm{~Pb}$ foi de $185 \pm 100 \mathrm{~Bq} \mathrm{~kg}^{-1}$ de solo seco, variando de 44 a $422 \mathrm{~Bq} \mathrm{~kg}^{-1}$ de solo seco, e na fração trocável (extraída com acetato de amônio $1 \mathrm{~N}$, a pH 7) só foi detectada em duas amostras, com atividades próximas de $6 \mathrm{~Bq} \mathrm{~kg}^{-1}$ de solo seco.

As atividades de ${ }^{210} \mathrm{~Pb}$ extraído na fração $\mathrm{F} 1$ (fração biodisponível) correlacionaram-se negativamente com o $\mathrm{pH}$ do solo, na profundidade amostrada $(\mathrm{r}=-0,82$; $\mathrm{p}=0,0124)$. Os resultados para ${ }^{237} \mathrm{U}$ e ${ }^{226} \mathrm{Ra}$ indicam possível solubilização de minerais de ${ }^{226} \mathrm{Ra}$ do solo e de particulados depositados na superfície e um teor mais elevado na fração F1. A mobilidade/biodisponibilidade do ${ }^{210} \mathrm{~Pb}$ tende a diminuir com o aumento do $\mathrm{pH}$ do solo e independe do teor total de ${ }^{210} \mathrm{~Pb}$ do solo (Langmuir, 1997).

As atividades de ${ }^{210} \mathrm{~Pb}$ na fase geoquímica $\mathrm{F} 2$ pouco variaram entre os cinco solos selecionados. A atividade média foi de $3 \mathrm{~Bq} \mathrm{~kg}^{-1}$ (Quadro 6). O percentual médio obtido para a fase F2 (redutível) foi de $2 \%$ do total. $\mathrm{Na}$ fase F3 (oxidável) esse percentual foi baixo e em torno de $1 \%$ para os cinco solos selecionados.

$\mathrm{Na} \mathrm{F} 4$, as atividades nos pontos 10, 42 e 46 foram muito próximas das observadas na F3. Entretanto, nos pontos 8 e 13 as atividades foram de 10 e $15 \mathrm{~Bq} \mathrm{~kg}^{-1}$, respectivamente. A textura mais argilosa verificada no Latossolo Amarelo distrófico, no ponto 8, e no Argissolo Vermelho-Amarelo, no ponto 13, deve ter influenciado nessas maiores atividades. A alta afinidade do ${ }^{210} \mathrm{~Pb}$ com os óxidos de Fe tem sido observada em vários trabalhos (Köhler et al., 2000; Ewers et al., 2003).

A fração residual F5 foi predominante, superando os $90 \%$ em todas as cinco áreas, com variação de 91 a $95 \%$ do teor de ${ }^{210} \mathrm{~Pb}$ total. Esses resultados demonstram que esse radionuclídeo está predominantemente associado à estrutura cristalina dos minerais do solo ou oclusa nos óxidos de $\mathrm{Fe}, \mathrm{Mn}$ e $\mathrm{Al}$ e apresenta 
biodisponibilidade muito baixa para os processos ecológicos. A alta atividade na F5 nos cinco solos selecionados demonstra que, provavelmente, o ${ }^{210} \mathrm{~Pb}$ adveio do material que deu origem a esses solos, e não de um processo de contaminação artificial em função da atividade da URA.

As atividades mais elevadas de ${ }^{238} \mathrm{U},{ }^{226} \mathrm{Ra}$ e ${ }^{210} \mathrm{~Pb}$ na fração geoquímica F1 nos solos mais ácidos, argilosos e relativamente próximos à usina, nos pontos 8 e 13, indicam uma possível solubilização de particulados oriundos da atividade da usina e um maior risco de contaminação da água subterrânea e transferência para cadeia alimentar.

\section{CONCLUSÕES}

1. As atividades totais e na fração geoquímica F1 de ${ }^{238} \mathrm{U}$ mais elevadas foram observadas nos solos mais ácidos e de textura mais argilosa. O ${ }^{238} \mathrm{U}$ apresentou biodisponibilidade média para processos ecológicos de aproximadamente $11 \%$ da atividade total e demonstrou ter afinidade pelos óxidos de Fe.

2. As atividades de ${ }^{226}$ Ra mais elevadas na fração geoquímica F1 (fração biodisponível) foram observadas nos solos de textura mais argilosa e ácidos. Os percentuais obtidos para ${ }^{226} \mathrm{Ra}$ na fração alcalina demonstraram que esse radionuclídeo tem pouca afinidade pelos óxidos de Fe.

3. O radionuclídeo ${ }^{210} \mathrm{~Pb}$ apresentou baixa biodisponibilidade, estando quase que em sua totalidade associado à fração residual, com percentual médio superior aos $90 \%$ da atividade total.

4. As atividades mais elevadas na fração geoquímica $\mathrm{F} 1$ de ${ }^{238} \mathrm{U},{ }^{226} \mathrm{Ra}$ e ${ }^{210} \mathrm{~Pb}$ ocorreram nos solos mais ácidos, argilosos e relativamente próximos à usina, indicando uma possível solubilização de particulados oriundos da atividade da usina.

5. As percentagens elevadas de ${ }^{238} \mathrm{U},{ }^{226} \mathrm{Ra} \mathrm{e}^{210} \mathrm{~Pb}$ na fração geoquímica F5 indicam que as atividades observadas nos cinco solos estão, predominantemente, associadas ao material que deu origem a eles, e não a um processo de contaminação artificial em função da atividade da URA.

\section{LITERATURA CITADA}

BAEZA, A.; PANIAGUA, J.M.; RUFO, M. \& BARANDICA, J. Bio-availability and transfer of natural radionuclides in a Mediterranean ecosystem. Appl. Radiat. Isotopes, 47:939945, 1996.

BOURG, A.C.M.; KEDZIOREK, M.A.M.; WASSERMAN, A. \& BOURG, I.C. Assessment of the mobility of heavy metals in soils and sediments. In: INTERNATIONAL SYMPOSIUM AND EXHIBITION ON ENVIRONMENTAL CONTAMINATION IN CENTRAL AND EASTERNEUROPE, 4., Warsaw, 1999. Proceedings. Warsaw, p.58-64. 1999.
BUNZL, K.; KRETNER, R.; SCHRAMEL, P.; SZELES, M. \& WINKLER, R. Speciation of ${ }^{238} \mathrm{U},{ }^{226} \mathrm{Ra},{ }^{210} \mathrm{~Pb},{ }^{228} \mathrm{Ra}$, and stable $\mathrm{Pb}$ in the soil near exhaust ventiling shaft of an uranium mine. Geoderma, 67:45-53, 1995.

BUTLER, A.P., CHEN, J., AGÜERO, A., EDLUND, O., ELERT, M., KIRCHNER, G., RASKOB, W., SHEPPARD, M., Performance assessment studies of models for water flow and radionuclide transport in vegetated soils using lysimeter data. J Environ Radioactivity 42, 271-288. 1999.

CARDOSO, G.V. Distribuição geoquímica e transferência solo-planta de radionuclídeos naturais em solos sob a influência de uma unidade de mineração e atividade de urânio. Seropédica, Universidade Federal Rural do Rio de Janeiro, 2004. 67p. (Tese de Mestrado)

DAVIS, P.A.; AVADHANULA, M.R.; CANCIO, D.; CARBONCRAS, P.; COUGHTREY, JOHANSSON, G.; LITTLE, R.H.; SMITH, G.M. \& WATKINS, B.M. BIOMOVS II: An international test of the performance of environmental transfer models. J. Environ. Radioact., 42:117-130, 1999.

EISENBUD, M. Environmental radioactivity. 3.ed. New York, Academic Press, 1987.

EMPRESA BRASILEIRA DE PESQUISA AGROPECUÁRIA EMBRAPA. Centro Nacional de Pesquisa do Solo. Manual de métodos de análise de solo. Rio de Janeiro, 1997. 212p.

EWERS, L.W.; HAM, G.J. \& WILKINS, B.T. Review of the transfer of naturally occurring radionuclides to terrestrial plants and domestic animals. National Radiological Protection Board. NRPB W, 49:2003. 70p.

GERZABEK, M.H.; STREBL, F. \& TEMMEL, B. Plant uptake of radionuclides in lysimeter experiments. Environ Pollut., 99:93-103, 1998.

INTERNATIONAL ATOMIC ENERGY AGENCY - IAEA. The application of the principles for limiting releases in the case of mining and milling of radioactive ores. Vienna, 1989. (IAEA Safety Series, 90)

INSTITUTO DE RADIOPROTEÇÃO E DOSIMETRIA - IRD/ DEPRA Manual de procedimentos analíticos do Departamento de Proteção Radiológica Ambiental. Rio de Janeiro, 1994. 52p.

KÖHLER, M.; GLEISBERG, B. \& NIESE, S. Investigation of the soil-plant transfer of primordial radionuclides in tomatoes by low-level $\gamma$-ray spectrometry. Appl. Radiat. Isotopes, 53:203-208, 2000.

LANGMUIR, D. Aqueous environmental geochemistry. New York, Printice-Hall, 1997. 600p.

LIMA, V.T. \& PENNA-FRANCA, E. Uptake of endogenous and exogenous ${ }^{226} \mathrm{Ra}$ by vegetation samples from soils of a highly radioactive region. Radiat. Prot. Dosim., 24:123126, 1998 .

MOLINARI, J. \& SNODGRASS, W.J. The chemistry and radiochemistry of radium and the other elements of the uranium and thorium natural decay series. In: The environmental behaviour of radium. Vienna, IAEA, 1990. p.11-56. (Technical Reports Series, 310) 
PRADO, V. C. DOS S. O impacto da produção de concentrado de urânio sobre a qualidade da água dos rios - Um estudo de caso na área do Complexo Mínero Industrial de Poços de Caldas. COPPE/UFRJ, RJ, 1994. 195p. (Tese Mestrado)

SCHULTZ, M.K.; INN, K.G.W.; LIN, Z.C.; BURNETT, W.C.; SMITH, G.; BIEGALSKI, S.R. \& FILLIBEN, J. Identification of radionuclide partitioning in soils and sediments: Determination of optimum conditions for the exchangeable fraction of the NIST standard sequential extraction protocol. Appl. Radiat. Isotopes, 49:1289-1293, 1998.

TESSIER, A.; CAMPBELL, P.G.C. \& BISSON, M. Sequential extraction procedure for the speciation of particulate trace metals. Anal. Chem., 51:844-851, 1979.

THIESSEN, K.M.; THORNE, M.C.; MAUL, P.R.; PRÖHL, G. \& WHEATER, H.S. Modeling radionuclide distribution and transport in the environment. Environ. Pollut., 100:151$177,1999$.

VASCONCELLOS, L.M.H.; AMARAL, E.C.S. \& VIANNA, M.E. Uptake of ${ }^{226} \mathrm{Ra}$ and ${ }^{210} \mathrm{~Pb}$ by food crops cultivated in a region of high natural radioactivity in Brasil. J. Environ. Radioact., 5:287-302, 1987.

WASSERMAN, M.A.; PEREZ, D.V. \& BOURG, A. Behavior of ${ }^{137} \mathrm{Cs}$ in some Brazilian Oxisols. Comm. Soil Sci. Plant, 33:1335-1349, 2002b.
WASSERMAN, M.A.; PEREZ D.V.; LAURIA, D.; SCHUCH, L.A. \& ZAGO, A. Valores de fator de transferência soloplanta do 226 Ra e 228Ra: Áreas agrícolas versus áreas de radioatividade natural elevada. In: ENCONTRO NACIONAL DE APLICAÇÕES NUCLEARES, 5., Rio de Janeiro, 2000. Anais. Rio de Janeiro, p. 48-53. 2000.

WASSERMAN, M.A.; CANNIZZARO, V.; KEDZIOREK, M.A.; WOLLER, A.; PFEIFFER, H.R. \& BOURG, A.C.M. Assessment of the potential mobility of anionic and cationic pollutants in contaminated soils. In: 2001 FALL MEETING, San Francisco, 2001b. H52C-0438.

WASSERMAN, M.A. \& LAURIA. D. Contribuição do aporte atmosférico na atividade de radionuclídeos naturais numa região rica em Monazita. In: ENCONTRO NACIONAL DE APLICAÇÕES NUCLEARES, 5., Rio de Janeiro, 2002. Anais. Rio de Janeiro, p.21-25. 2002.

WASSERMAN, M.A.; PEREZ, D.V.; BARTOLY, F. \& POQUET, I. Assessment of the mobility and bioavailability of $60 \mathrm{Co}$ and $137 \mathrm{Cs}$ in contaminated soils. In: REGIONAL CONGRESS ON RADIATION PROTECTION AND SAFETY, 5, Recife, 2001. Proceedings. Recife, p.63-71. 2001a.

WASSERMAN, M.A.; PEREZ, D.V.; CONTI, C.C.; BARTOLY, F.; VIANA, A.G.; WASSERMAN, J.C. \& MOURA, G.P. Transfer and mobility of ${ }^{137} \mathrm{Cs}$ and ${ }^{60} \mathrm{Co}$ in Oxisol and Alfisol. R. Bras. Pesq. Desenvol., 4:737-741, 2002a. 